\title{
Multiplicative Error Modeling Approach for Time Series Forecasting
}

\author{
Poulami Chowdhury \\ Department of Statistics \\ Presidency University \\ Kolkata, India \\ poulami.chowdhury3@gmail.com
}

\author{
Tanujit Chakraborty \\ $S Q C \& O R$ Unit \\ Indian Statistical Institute \\ Kolkata, India \\ tanujit_r@isical.ac.in
}

\begin{abstract}
Real-world time series data sets contain a combination of linear and nonlinear patterns, making the time series forecasting problem more challenging. In this paper, a new hybrid methodology is introduced for forecasting univariate time series data sets using a multiplicative error modeling approach. An autoregressive integrated moving average (ARIMA) model is combined with an autoregressive neural network (ARNN) for improving the predictions of individual forecast models. The proposed multiplicative ARIMA-ARNN model glorifies the chances of capturing the different combinations of linear and nonlinear patterns in time series. The model shows outstanding performance on six standard time-series data sets compared to other widely used single and hybrid forecasting models.
\end{abstract}

Index Terms-Multiplicative error, ARIMA, Neural net.

\section{INTRODUCTION}

Time series forecasting plays a vital role in understanding social, economic, epidemiological, and organizational behavior. The univariate forecasting models are handy when very little knowledge is available on the underlying data generating process or when there is no satisfactory explanatory model that relates the prediction variable to other explanatory variables. The traditional stochastic time series model, for example, autoregressive integrated moving average (ARIMA), is highly useful to forecast linear time series data [1]. In contrast, the autoregressive neural network (ARNN) model performed superior in modeling nonlinear time series data [2]. Also, modern machine learning tools such as artificial neural networks (ANNs), support vector machines (SVM) have found an increasing consideration in forecasting theory [3].

Since the time series data sets contain both linear and nonlinear patterns, making decisions based on an individual model is critical. Undoubtedly, a single model is not sufficient to deal with complicated real-world systems with unknown mixed patterns. By hybridizing linear and nonlinear models, one can reduce the bias and variances of the prediction error of component models [4]. Thus, a combination of both the linear and nonlinear models will be preferred to model such complex autocorrelation structures accurately. Several hybrid models based on additive error modeling approach were applied in the past to solve various forecasting problems that arose in the stock market, financial econometrics, electricity, epidemiology, and other applied areas [5]-[9]. But these hybrid models as- sume an additive relationship between the linear and nonlinear components of the time series.

Motivated by this, we propose a multiplicative ARIMAARNN model which considers interactions (multiplicative relationships) between the linear and nonlinear components of a time series. In the first phase of the proposed model, an ARIMA model is applied to catch the linear patterns of the data set. Multiplicative error values of the ARIMA model are calculated and restored for further modeling. In the second stage, a nonlinear ARNN model is applied to model the unexplained nonlinearities in the residual series. The proposed multiplicative error modeling approach will be more appropriate for use when measuring the rate of change in errors instead of measuring absolute changes in previously used additive hybrid models. For example, if we consider unemployment, accident, traffic, etc. data sets, the multiplicative ARIMA-ARNN model is expected to perform better over Zhang's hybrid (additive) ARIMA-ANN model [5]. The proposed two-step approach is appropriate for better explaining nonlinearity and nonstationarity in the data. An application of the proposed methodology is shown on six standard data sets.

The rest of the paper is organized as follows. In section 2 , we discuss the constituent methods to be used in the proposed model. In Section 3, we describe the formulation of the proposed multiplicative ARIMA-ARNN model. Section 4 is entirely devoted to the experimental evaluation of six distinct publicly available data sets from various applied fields. Finally, Section 5 concludes the paper with some future scope of research.

\section{BACKGROUND}

This work proposes a multiplicative ARIMA-ARNN model for time series forecasting. Before describing the proposed methodology, we briefly describe the individual models to be used in the hybridization.

\section{A. ARIMA Model}

ARIMA is a popular linear time series model in classical time series forecasting literature. In an autoregressive integrated moving average model, the future values of a variable are assumed to be a linear function of several past 
observations and random errors. We denote an ARIMA model by $\operatorname{ARIMA}(p, d, q)$ with parameters $p, q$, and $d$ are the order of the autoregressive model, the order of the moving average model and the value of the differencing term, respectively [1]. The mathematical formulation of an ARIMA model is as follows:

$$
Y_{t}=\mu+\sum_{i=1}^{p} \phi_{i} Y_{t-i}+\varepsilon_{t}-\sum_{j=1}^{q} \theta_{j} \varepsilon_{t-j},
$$

where $Y_{t}$ and $\varepsilon_{t}$ denote the actual value of the time series and random error at time $t$, respectively; $\mu$ is a constant and $\phi_{i}$ and $\theta_{j}$ are the coefficients of the model. To construct an ARIMA model for a time series data, one requires to follow three basic iterative steps: (a) identification of model (achievingstationarity), (b) estimation of model parameters (the 'best' choices of $p$ and $q$ ) and (c) checking model diagnostics (finding the 'best' fitted ARIMA model using akaike's information criterion (AIC)).

\section{B. ARNN Model}

Neural nets, the most popular machine learning model for supervised learning, are based on mathematical modeling of the human brain. Any standard neural network architecture comprises of a sequential organization of layers as a network of "neurons". The predictors (or inputs), intermediate layers (or hidden layer) and the forecasts (or outputs) are organized together in a feedforward neural network model. The forecasts can be obtained as a linear combination of the inputs and weights are attached with the predictors. For time series forecasting, ARNN model is built using the lagged values (inputs to neural net) of the time series data [2]. The $\operatorname{ARNN}(m, k)$ model applies a one-hidden-layer feedforward neural network with $m$ lagged inputs and $k$ nodes in its hidden layer. The mathematical formulation of the ARNN model is as follows:

$$
\hat{Z}_{t}=\psi_{0}\left\{w_{c_{0}}+\sum_{k} w_{k_{0}} \psi_{k}\left(w_{c_{k}}+\sum_{i} w_{i_{k}} Z_{t-j_{i}}\right)\right\},
$$

where $\left\{Z_{t-j_{i}}: j=1,2, \cdots, m\right\}$ be the inputs, $\left\{w_{c_{k}}\right\}$ be the connecting weights, and $\psi_{i}$ is the logistic sigmoidal activation function. The connection weights are trained using standard gradient descent backpropagation [3]. The $\operatorname{ARNN}(m, k)$ model uses $p$ as the number of lags for an $\operatorname{AR}(m)$ model and $k$ is usually set to $k=\left[\frac{m+1}{2}\right]$ for non-seasonal time series data [3].

\section{MULTIPLICATIVE ARIMA-ARNN MODEL}

Multiplicative ARIMA-ARNN model is a two-step method. At first, an ARIMA model is built to model the linear components of a given time series, and a series of forecasts are generated. In the second stage, the ARIMA residual (multiplicative error) series are modeled using a nonlinear ARNN model. The previous studies on hybrid time series models based on linear and nonlinear time series assume the additive error structures [5], [7]-[9]. We considered the multiplicative error structures and proposed a multiplicative model based on ARIMA and ARNN. Below we present the formulation of the proposed model (also see Figure 1):

$$
X_{t}=Y_{t} \times Z_{t},
$$

where $Y_{t}$ is the linear part, and $Z_{t}$ is the nonlinear part of the proposed model. We can estimate both $Y_{t}$ and $Z_{t}$ from the training data set. Let, $\hat{Y}_{t}$ be the forecast value of the ARIMA model at time $\mathrm{t}$ and $\varepsilon_{t}$ represent the error residuals at time $\mathrm{t}$, obtained from the ARIMA model. We can then write

$$
\varepsilon_{t}=X_{t} / \hat{Y}_{t} .
$$

The multiplicative residuals will be modeled by the ARNN model and can be represented as follows

$$
\varepsilon_{t}=f\left(\varepsilon_{t-1}, \varepsilon_{t-2}, \ldots, \varepsilon_{t-n}\right)+\varsigma_{t},
$$

where $f$ is a nonlinear function, and the modeling is done by the ARNN model and $\varsigma_{t}$ is the random shocks. Therefore, we can write the combined forecast as:

$$
\hat{X}_{t}=\hat{Y}_{t} \times \hat{Z}_{t},
$$

where $\hat{Z}_{t}$ is the forecasted value of the ARNN model. The ARNN model is used to remodel the left-over autocorrelations in the residuals, which ARIMA could not model. The proposed model can be looked upon as an ARIMA error remodeling approach. This is important because the linear ARIMA model may fail to generate white noise behavior for the forecast residuals due to model misspecification and disturbances in the economic and business time series. Therefore, if the error series is modeled properly, the performance of the original forecaster can be improved, even though marginally at times.

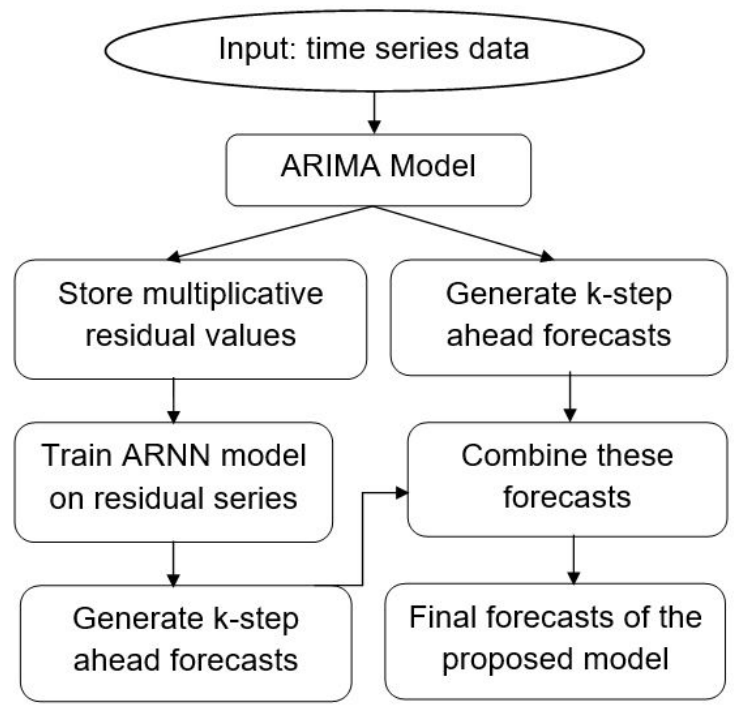

Fig. 1. Flow diagram of the proposed multiplicative ARIMA-ARNN model

It is important to note that the multiplicative model concept is more useful than the additive approach because it considers the interactions between linear and nonlinear components of the time series. Additive models separately model the 
TABLE I

DESCRIPTIONS OF SIX STANDARD TIME SERIES DATA SETS

\begin{tabular}{|c|c|c|c|}
\hline Data & Training size & Validation size & Test size \\
\hline Accidental Death & 43 & 14 & 15 \\
Canadian Unemployment & 144 & 48 & 48 \\
Chickenpox & 298 & 100 & 100 \\
Passenger & 86 & 29 & 29 \\
Traffic & 108 & 36 & 36 \\
Wolf's Sunspot & 172 & 58 & 58 \\
\hline
\end{tabular}

linear and nonlinear parts where the proposed multiplicative hybridization approach is more appropriate for economic and business data sets since there may be interactions between linear and nonlinear components for these nonstationary and nonlinear time series.

\section{EXPERIMENTAL ANALYSIS}

In this section, six open-access time series data sets, namely Accidental death (USA), Canadian unemployment, Chickenpox, Airline Passenger, Traffic, and Wolf's sunspot data are used to determine the effectiveness of the proposed model. These data sets display different patterns of behavior and taken from various applied fields for experimentation.

\section{A. Data}

Six standard time series data sets were collected from openaccess time series data repositories [3]. Accidental death data set contains monthly accidental deaths in the USA from 1973 to 1978 , whereas the Canadian unemployment data set has monthly total unemployment figures (in thousands) from 1956 to 1975 . Another data set is the monthly reported number of chickenpox in New York City from 1931 to 1972 . The passenger data set comprises of monthly totals from International airport passengers from 1949 to 1960 . Traffic data set contains monthly traffic facilities in Ontario from 1960 to 1974. Lastly, Wolf's sunspot data set is considered, taken from the year 1700 to 1987 , contains yearly sunspot numbers. A brief summary of the data sets are given in Table I.

\section{B. Performance metrics}

Performance metrics such as root mean square error (RMSE) and Symmetric Mean Absolute Percent Error (SMAPE) are used to evaluate the performances of different forecasting models for the above time series data sets [1].

$$
\begin{aligned}
R M S E & =\sqrt{\frac{1}{n} \sum_{i=1}^{n}\left(y_{i}-\hat{y}_{i}\right)^{2}} \\
S M A P E & =\frac{1}{n} \sum_{i=1}^{n} \frac{\left|y_{i}-\hat{y}_{i}\right|}{\left(\left|y_{i}\right|+\left|\hat{y}_{i}\right|\right) / 2}
\end{aligned}
$$

where $y_{i}$ is the actual value, $\hat{y}_{i}$ is the predicted output, and $n$ denotes the number of data points. The lower the value of these performance metrics, the better is the performance of the forecasting model.

\section{Results}

Six-time series data sets are divided into training (60\% of the data set), validation (20\% of the data set) and testing data (20\% of the data set). These data sets are mostly nonlinear and non-gaussian in nature. In this section, we experimentally evaluate the performances of ARIMA, ANN, SVM, ARNN model, hybrid ARIMA-ANN model [5], hybrid ARIMA-SVM model [10], hybrid ARIMA-ARNN model [8] in comparison with the proposed multiplicative ARIMA-ARNN model for all these data sets.

We start the experimental evaluation with the classical $\operatorname{ARIMA}(p, d, q)$ using the "forecast" package in $\mathrm{R}$ statistical software. We first performed a unit root test for stationarity check for the model to determine the value of $d$ in the ARIMA model. The 'best' fitted ARIMA model is chosen using AIC value for each training data sets. As the ARIMA model is fitted, predictions are generated for one year and three year time steps. We also compute training data predicted values and calculate the residual errors. In the second stage, residuals obtained using the ARIMA model are remodeled with $\operatorname{ARNN}(m, k)$ model. We employ a pre-defined Box-Cox transformation set to $\lambda=0$ to ensure the forecast values to stay positive. The value of $m$ and $k$ is obtained by training the network, which is a data-dependent approach as in [3]. Further, both the linear and nonlinear forecasts are multiplied together to get the final estimates. Other individual models like SVM was implemented using the "e1071" package in R statistical software. ARNN was performed using the "nnfor" package with the "nnetar" function, and for the ANN model, we have used the "caret" package with "mlp" function in $\mathrm{R}$ statistical software.

The experimental results are obtained as follows: ARIMA $(2,0,2)$ was fitted to accidental death data set with AIC and loglikelihood $(L)$ values -269.53 and 100.78 , respectively. The ARIMA residuals (multiplicative) were further trained with ARNN $(6,3)$ model with an average of 20 networks. Further, we computed the predicted test outputs of the multiplicative ARIMA-ARNN model and compared it with actual test outputs. The values of performance metrics are reported in Table III and II. For the Canadian unemployment data set, $\operatorname{ARIMA}(2,0,3)$ having AIC $=-395.12$ and $L=152.73$ was fitted. An ARNN $(3,2)$ model (3-2-1 network configuration) with an average of 20 networks, each having ten weights, was trained on ARIMA-given multiplicative residuals. Both the forecasted results of ARIMA and ARNN are multiplied together to obtain the final forecast values, and performance measures are calculated and reported in Table III and II. $\operatorname{ARIMA}(4,0,2)$ was fitted to Chickenpox data having AIC $=-550.34$ and $\log$-likelihood $(L)$ value as 278.12. Further, the model's multiplicative residuals were trained using ARNN $(12,6)$ model with an average of 20 networks, each of which is a 12-6-1 network with 112 weights. Then the forecast results of ARIMA along with ARNN residual forecasts are multiplied together to obtain the final forecast values, and values of RMSE and SMAPE are reported in Table III and 
TABLE II

RMSE VALUES FOR DIFFERENT FORECASTING MODELS ON SIX STANDARD TIME SERIES DATA SETS (BEST VALUES IN BOLD)

\begin{tabular}{|c|c|c|c|c|c|c|c|c|}
\hline Model & ARIMA & ANN & ARNN & SVM & $\begin{array}{c}\text { Hybrid } \\
\text { ARIMA-SVM [10] }\end{array}$ & $\begin{array}{c}\text { Hybrid } \\
\text { ARIMA-ANN [5] }\end{array}$ & $\begin{array}{c}\text { Hybrid } \\
\text { ARIMA-ARNN [8] }\end{array}$ & $\begin{array}{c}\text { Multiplicative } \\
\text { ARIMA-ARNN }\end{array}$ \\
\hline Accidental Death & 379.06 & 390.13 & 395.44 & 415.21 & 285.12 & $\mathbf{2 7 9 . 5 7}$ & 280.59 \\
\hline Canadian Unemployment & 53.372 & 46.160 & 43.988 & 50.286 & 47.788 & 43.712 & 40.902 \\
\hline Chickenpox & 229.45 & 157.45 & 149.12 & 170.12 & 151.02 & 145.75 & 140.96 & $\mathbf{3 4 . 4 2 5}$ \\
\hline Passenger & 36.456 & 33.489 & 26.780 & 32.120 & 26.120 & 25.702 & 24.900 \\
\hline Traffic & 31.743 & 24.456 & 23.045 & 29.549 & 23.123 & 21.032 & $\mathbf{1 2 5 . 0 3}$ \\
\hline Wolf's Sunspot & 31.052 & 29.145 & 24.178 & 29.453 & 22.018 & 23.489 & 23.060 \\
\hline
\end{tabular}

TABLE III

SMAPE VALUES FOR DIFFERENT FORECASTING MODELS ON SIX STANDARD TIME SERIES DATA SETS (BEST VALUES IN BOLD)

\begin{tabular}{|c|c|c|c|c|c|c|c|c|}
\hline Model & ARIMA & ANN & ARNN & SVM & $\begin{array}{c}\text { Hybrid } \\
\text { ARIMA-SVM [10] }\end{array}$ & $\begin{array}{c}\text { Hybrid } \\
\text { ARIMA-ANN [5] }\end{array}$ & $\begin{array}{c}\text { Hybrid } \\
\text { ARIMA-ARNN [8] }\end{array}$ & $\begin{array}{c}\text { Multiplicative } \\
\text { ARIMA-ARNN }\end{array}$ \\
\hline Accidental Death & 7.3260 & 5.9112 & 4.8531 & 7.1530 & 4.6011 & $\mathbf{3 . 4 9 6 6}$ & 4.0593 \\
\hline Canadian Unemployment & 7.1133 & 6.9509 & 6.6000 & 7.9560 & 6.1231 & 6.4606 & 3.8663 \\
\hline Chickenpox & 24.073 & 24.048 & 23.563 & 24.393 & 19.818 & 17.775 & 17.2069 \\
\hline Passenger & 9.9666 & 6.8823 & 6.2035 & 7.1521 & 4.7014 & 5.9366 & 4.9670 & $\mathbf{4 . 7 9 0 4}$ \\
\hline Traffic & 18.381 & 17.966 & 13.117 & 18.154 & 16.117 & 12.459 & $\mathbf{1 7 . 4 3 0}$ \\
\hline Wolf's Sunspot & 8.3699 & 4.8140 & 4.1193 & 4.2227 & 4.4400 & 4.4244 & 4.294 & 13.053 \\
\hline
\end{tabular}

II. For Passenger data, $\operatorname{ARIMA}(3,1,3)$ was fitted with AIC $=$ -86.12 and $L=43.18$. Further, ARIMA residuals were trained using ARNN(16,8) model (16-8-1 network configuration) with an average of 20 networks, each having 145 weights and final forecasts of multiplicative ARIMA-ARNN model is obtained. We report the values of the performance metrics in Table III and II.

Finally, ARIMA $(1,1,1)$ with AIC $=-1248.46$ and $L$ equals 781.23 is fitted to the Traffic data set whereas $\operatorname{ARIMA}(2,0,1)$ was fitted to the Wolf's Sunspot series having AIC $=-45.12$ and $L=12.89$. The residuals (multiplicative) are obtained from the ARIMA model for these two data sets were trained using ARNN $(3,2)$ model with an average of 20 networks, each of which is a 3-2-1 network with 11 weights. Finally, the forecast results of ARIMA and ARNN residual forecasts are multiplied together to obtain the predicted forecasted values. We then compute the values of the performance measures and report them in Table III and II.

For comparison purposes, we applied single ARIMA, ANN, ARNN, SVM, along with hybrid ARIMA-ANN [5], hybrid ARIMA-SVM model [10] and hybrid ARIMA-ARNN model [8] models to these time-series data sets. The predicted forecasts for the test data sets of the proposed hybrid model for six standard time series data sets are used for model adequacy checking and based on actual and predicted test outputs we computed RMSE and SMAPE for all the data sets (also see Table III and II). The performances of the proposed multiplicative ARIMA-ARNN model is superior as compared to all the individual models. Compared to the state-of-the-art hybrid models, the proposal outperformed all the state-of-theart hybrid models in a significant margin for four data sets.

\section{DISCUSSION}

A new multiplicative ARIMA-ARNN model using multiplicative error modeling is proposed in this paper. The proposed model performs superior for four out of six publicly available data sets. The proposed multiplicative ARIMAARNN model filters out linearity using the ARIMA model and predicts nonlinearities present in the error residuals (multiplicative) with the ARNN model. The proposed multiplicative model is better than the additive ones since it considers the interactions between the linear and nonlinear components of the time series. Since real-world time series data sets often fluctuate heavily, the multiplicative ARIMA-ARNN model will be a 'good' alternative for time series practitioners. An immediate future work of this work will be to improve this model for multivariate time series forecasting scenario.

\section{REFERENCES}

[1] G. E. Box, G. M. Jenkins, G. C. Reinsel, and G. M. Ljung, Time series analysis: forecasting and control. John Wiley \& Sons, 1970.

[2] J. Faraway and C. Chatfield, "Time series forecasting with neural networks: a comparative study using the air line data," Journal of the Royal Statistical Society: Series C (Applied Statistics), vol. 47, no. 2, pp. 231-250, 1998.

[3] R. J. Hyndman and G. Athanasopoulos, Forecasting: principles and practice. OTexts, 2018.

[4] M. R. Oliveira and L. Torgo, "Ensembles for time series forecasting," J. Mach. Learn. Res., vol. 39, pp. 360-370, 2014.

[5] G. P. Zhang, "Time series forecasting using a hybrid arima and neural network model," Neurocomputing, vol. 50, pp. 159-175, 2003.

[6] C. H. Aladag, E. Egrioglu, and C. Kadilar, "Forecasting nonlinear time series with a hybrid methodology," Applied Mathematics Letters, vol. 22, no. 9, pp. 1467-1470, 2009.

[7] M. Khashei and Z. Hajirahimi, "A comparative study of series arima/mlp hybrid models for stock price forecasting," Communications in StatisticsSimulation and Computation, pp. 1-16, 2018.

[8] T. Chakraborty, S. Chattopadhyay, and I. Ghosh, "Forecasting dengue epidemics using a hybrid methodology," Physica A: Statistical Mechanics and its Applications, vol. 527, 2019.

[9] T. Chakraborty and I. Ghosh, "Real-time forecasts and risk assessment of novel coronavirus (covid-19) cases: A data-driven analysis," Chaos, Solitons and Fractals, vol. 135, 2020.

[10] C. Ordóñez, F. S. Lasheras, J. Roca-Pardiñas, and F. J. de Cos Juez, “A hybrid arima-svm model for the study of the remaining useful life of aircraft engines," Journal of Computational and Applied Mathematics, vol. 346, pp. 184-191, 2019. 